\title{
Diversidade e flutuação populacional de ácaros (Acari) em amora-preta (Rubus fruticosus, Rosaceae) no estado do Rio Grande do Sul, Brasil
}

\author{
Márla Maria Marchetti \& Noeli Juarez Ferla
}

Museu de Ciências Naturais, Centro Universitário UNIVATES, Avenida Avelino Tallini, 171, 95900-000 Lajeado, RS, Brasil. (mmmarla_acaro@yahoo.com.br; njferla@univates.br)

\begin{abstract}
Diversity and population fluctuation of mites (Acari) in blackberry (Rubus fruticosus, Rosaceae) in the state of Rio Grande do Sul, Brazil. Mites of the families Eriophyidae, Tarsonemidae and Tetranychidae are considered of economic importance in the blackberry (Rubus fruticosus, Rosaceae), raspberry and other small fruit crops. This study was conducted from November of 2001 to October of 2003 on Brazos, Caigangue and Tupy cultivars in Ilópolis municipality, state of Rio Grande do Sul, to describe the mitefauna and its population fluctuation in blackberry crops. A total of 36,094 mites belonging to 12 families was collected. Most of the mites belong to Diptilomiopidae (80.9\%) and Tetranychidae (13.9\%), composed predominantly of phytophagous species. Chakrabartiella sp. (Diptilomiopidae) was the most abundant species, followed by Neotetranychus asper Feres \& Flechtmann, 2000 (Tetranychidae). Stigmaeidae (2.1\%) and Phytoseiidae (0.4\%) were the most common families of predaceous mites, and Agistemus brasiliensis Matioli, Ueckermann \& Oliveira, 2002 (Stigmaeidae) and Typhlodromalus aripo DeLeon, 1967 (Phytoseiidae) the most common predaceous species.
\end{abstract}

KEYWORDS. Agroecossystems, Diptilomiopidae, Tetranychidae, Phytoseiidae, Stigmaeidae.

RESUMO. Ácaros das famílias Eriophyidae, Tarsonemidae e Tetranychidae são citados como de importância econômica na cultura de amora-preta (Rubus fruticosus, Rosaceae), framboesa e outras frutas pequenas. Este estudo foi conduzido de novembro de 2001 a outubro de 2003 nas cultivares Brazos, Caigangue e Tupy no município de Ilópolis, Rio Grande do Sul, com o objetivo de conhecer a acarofauna e sua dinâmica populacional em amora-preta. Foram coletados 36.094 ácaros pertencentes a 11 famílias. A maioria dos ácaros coletados pertence às famílias Diptilomiopidae $(80,9 \%)$ e Tetranychidae $(13,9 \%)$, compostas por espécies fitófagas. Chakrabartiella sp. (Diptilomiopidae), foi a espécie mais abundante, seguida por Neotetranychus asper Feres \& Flechtmann, 2000 (Tetranychidae). Stigmaeidae $(2,1 \%)$ e Phytoseiidae $(0,4 \%)$ foram os ácaros predadores mais comuns, sendo Agistemus brasiliensis Matioli, Ueckermann \& Oliveira, 2002 (Stigmaeidae) e Typhlodromalus aripo DeLeon, 1967 (Phytoseiidae), as espécies de predadores mais abundantes.

PALAVRAS-CHAVE. Agroecossistemas, Diptilomiopidae, Tetranychidae, Phytoseiidae, Stigmaeidae.

A amora-preta (Rubus fruticosus, Rosaceae) é cultivada em quase todas as regiões do mundo, devido a grande capacidade de adaptação a diferentes condições climáticas. No Rio Grande do Sul, principal produtor brasileiro, a maior produção encontra-se nos municípios de Feliz e Vacaria, onde a cultivar Tupy responde por $70 \%$ da área cultivada (ANTUNEs et al., 2002).

Esta cultura é atacada por pragas e doenças (BASSOLS, 1980; RASEIRA et al., 1984). Ácaros das famílias Eriophyidae, Tarsonemidae e Tetranychidae são citados como de importância econômica na cultura de amora-preta (JePpson et al., 1975; AmRine \& Stasny, 1994; De Lillo \& Duso, 1996). Os eriofídeos são os principais ácaros registrados nesse cultivo, sendo Phyllocoptes gracilis (Nalepa, 1890) e Acalitus essigi (Hassan, 1928) os mais importantes (De Lillo \& Duso, 1996). Phyllocoptes gracilis produz manchas cloróticas nas folhas, podendo causar necrose, enquanto altas infestações provocam deformação e enrolamento das mesmas. Infestações contínuas diminuem o vigor das plantas e a qualidade dos frutos. Acalitus essigi provoca o amadurecimento irregular dos frutos e, em altas infestações, os frutos maduros podem apresentar cor vermelha (JEPPSON et al., 1975; De Lillo \& Duso, 1996).

Várias espécies de tetraniquídeos são citadas nesta cultura (Bolland et al., 1998), destacando-se Neotetranychus rubi Trägardh, 1915. Amoreiras também podem ser atacadas por Tetranychus urticae Koch, 1836 e T. mexicanus (McGregor, 1950). Estas espécies ocupam a face inferior das folhas e determinam o aparecimento de cloroses; as folhas ficam amareladas, secam e caem prematuramente, reduzindo a produção (FLECHTMANN, 1979).

Devido à importância econômica desta cultura, estudos que oportunizem conhecer os ácaros fitófagos e predadores associados são relevantes. A flutuação de ácaros varia dependendo da época do ano, do local e do hospedeiro. Por conseguinte, o conhecimento da flutuação populacional e da época de maior ocorrência de uma determinada espécie de ácaro de importância econômica é um requisito indispensável para o estabelecimento de um controle eficiente e racional, permitindo planejar estratégias de manejo mais eficazes. O objetivo deste estudo foi conhecer a fauna acarina e sua dinâmica populacional em três cultivares de amorapreta, no município de Ilópolis, Rio Grande do Sul.

\section{MATERIAL E MÉTODOS}

Este estudo foi conduzido em um campo de amorapreta de 2 ha no município de Ilópolis, Rio Grande do Sul ( $28^{\circ} 55^{\prime} 43,39^{\prime \prime} \mathrm{S}, 52^{\circ} 7$ ' 37,51”W), com as cultivares Brazos, Caigangue e Tupy distribuídas em quatro fileiras em cada cultivar separadas por aproximadamente $5 \mathrm{~m}$ entre as fileiras. As amostragens foram realizadas entre os meses de novembro de 2001 e outubro de 2003. 
De cada cultivar foi coletado mensalmente, e de forma aleatória, 90 folíolos. Os folíolos foram individualizados em sacos de papel, guardados em caixa de isopor com gelo reutilizável e transportados ao laboratório para realização da contagem e a coleta dos ácaros. A contagem foi realizada diretamente sobre os folíolos da amoreira utilizando microscópio estereoscópico, observando as duas faces dos folíolos. Os ácaros coletados foram mantidos em álcool etílico a $70 \%$ e posteriormente todos montados usando o meio de Hoyer (Zhang, 2003), exceto os Diptilomiopidae que foram montados em meio de Berlese modificado (AMRINE \& MAnson, 1996). As lâminas foram mantidas em estufa a $50-60^{\circ} \mathrm{C}$ por cerca de 10 dias para a fixação, distensão e clarificação dos espécimes e secagem do meio.

$\mathrm{O}$ índice de riqueza das espécies de Margalef (R), equitabilidade de Pielou (E), Shannon-Wiener (H') e diversidade máxima teórica (H max) foram calculados utilizando o programa estatístico DivEs (RoDRIGUES, 2005). Os parâmetros climáticos foram obtidos da Estação Climatológica da Embrapa Uva e Vinho, município de Bento Gonçalves, Rio Grande do Sul.

Espécimes representantes de cada uma das espécies encontradas foram depositados na coleção de referência de ácaros, do Museu de Ciências Naturais do Centro Universitário UNIVATES, Lajeado, Rio Grande do Sul.

\section{RESULTADOS E DISCUSSÃO}

Foram coletados 36.094 ácaros de 27 morfoespécies pertencentes a 12 famílias (Tab. I) (cerca de 99,3\% dos ácaros pertencente à subordem Actinedida). Do total de espécimes coletados, $78,1 \%$ foram encontrados na cultivar Caigangue, $11,3 \%$ na cultivar Brazos e 10,5\% na
Tupy. O esforço amostral foi suficiente, pois a curva do coletor encontrou a estabilidade em todas as cultivares em agosto de 2003, após vinte e quatro coletas (Fig. 1).

Abundância da acarofauna. A maioria dos ácaros esteve representada por Diptilomiopidae, Tenuipalpidae e Tetranychidae, reconhecidos como fitófagos. Diptilomiopidae representou 80,9\% dos ácaros coletados no levantamento, sendo Chakrabartiella sp., a única espécie registrada. Na cultivar Caigangue, 90,4\% dos ácaros coletados pertenceram a esse gênero, enquanto que nas cultivares Brazos e Tupy as proporções foram de 48,6\% e 44,5\%, respectivamente. Segundo AmRInE \& STASNY (1994), C. ficusis (Chakrabarti, Ghosh \& Das, 1992), a única espécie do gênero, foi registrada somente sobre Ficus sp., na Índia. Portanto, essa é a primeira citação desse gênero sobre Rubus sp. ainda que várias espécies de diptilomiopídeos tenham sido citadas em diferentes gêneros de Rosaceae (OLDFIELD, 1996).

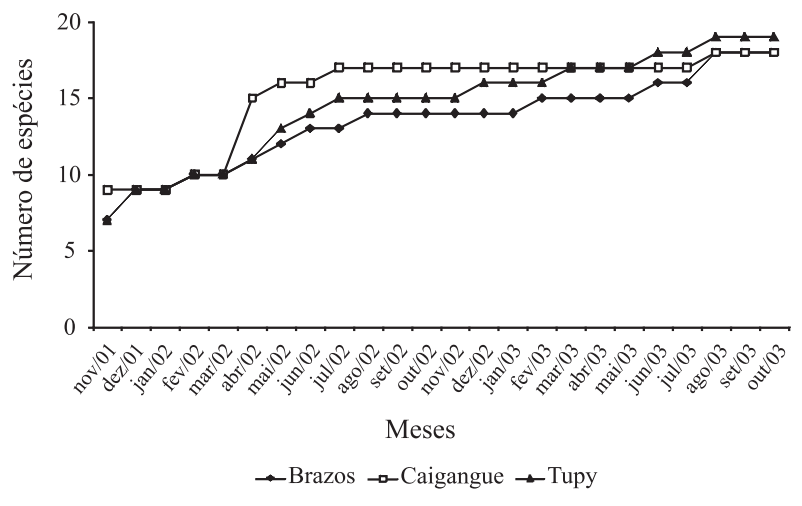

Figura 1. Curva do coletor mostrando o esforço amostral realizado nas cultivares Brazos, Caigangue e Tupy de amora-preta (Rubus fruticosus, Rosaceae), em Ilópolis, Rio Grande do Sul, entre os meses de novembro de 2001 a outubro de 2003.

Tabela I. Acarofauna coletada em amora-preta (Rubus fruticosus, Rosaceae) nas cultivares Brazos, Caigangue e Tupy, no município de Ilópolis, Rio Grande do Sul, entre os meses de novembro de 2001 a outubro de 2003.

\begin{tabular}{|c|c|c|c|c|c|c|c|c|c|c|}
\hline Subordens & Famílias & Espécies & Brazos & $\%$ & Caigangue & $\%$ & Tupy & $\%$ & Total & $\%$ \\
\hline Acaridida & Acaridae & Tyrophagus putrescentiae & & & & & 2 & 0,05 & 2 & 0,005 \\
\hline \multirow[t]{13}{*}{ Actinedida } & Bdellidae & Bdellodes sp. & & & 1 & 0,003 & & & 1 & 0,002 \\
\hline & Cunaxidae & Cunaxoides sp. & 14 & 0,3 & 34 & 0,1 & 17 & 0,4 & 65 & 0,1 \\
\hline & Diptilomiopidae & Chakrabartiella sp. & 1.987 & 48,6 & 25.528 & 90,4 & 1.696 & 44,5 & 29211 & 80,9 \\
\hline & Stigmaeidae & Agistemus brasiliensis & 168 & 4,1 & 430 & 1,5 & 163 & 4,2 & 761 & 2,1 \\
\hline & Tarsonemidae & Tarsonemus sp. & & & 6 & 0,02 & 18 & 0,4 & 24 & 0,06 \\
\hline & & Xenotarsonemus sp. & 14 & 0,3 & 23 & 0,08 & 2 & 0,05 & 39 & 0,1 \\
\hline & Tenuipalpidae & Brevipalpus phoenicis & 24 & 0,5 & 103 & 0,3 & 5 & 0,1 & 132 & 0,3 \\
\hline & Tetranychidae & Eotetranychus sp. & 58 & 1,4 & 49 & 0,1 & & & 107 & 0,2 \\
\hline & & Neotetranychus asper & 1.676 & 41,0 & 1.817 & 6,4 & 1.374 & 36,1 & 4867 & 13,4 \\
\hline & & Oligonychus yothersi & 31 & 0,7 & 45 & 0,1 & 20 & 0,5 & 96 & 0,2 \\
\hline & & Tetranychus mexicanus & & & 3 & 0,01 & & & 3 & 0,01 \\
\hline & Iolinidae & Pronematus anconai & 3 & 0,07 & 7 & 0,02 & & & 10 & 0,02 \\
\hline & Tydeidae & Tydeus sp. & 72 & 1,7 & 15 & 0,05 & 452 & 11,8 & 539 & 1,4 \\
\hline \multirow[t]{12}{*}{ Gamasida } & Parasitidae & Parasitus sp. & & & & & 1 & 0,02 & 1 & 0,002 \\
\hline & Phytoseiidae & Amblyseius compositus & & & & & 5 & 0,1 & 5 & 0,001 \\
\hline & & Amblyseius herbicolus & & & & & 1 & 0,02 & 1 & 0,002 \\
\hline & & Amblyseius neochiapensis & 1 & 0,02 & 1 & 0,003 & & & 2 & 0,005 \\
\hline & & Euseius ho & 6 & 0,1 & & & 14 & 0,3 & 20 & 0,05 \\
\hline & & Galendromus (G.) annectens & 4 & 0,09 & 3 & 0,01 & & & 7 & 0,01 \\
\hline & & Metaseiulus (M.) camelliae & & & & & 1 & 0,02 & 1 & 0,002 \\
\hline & & Neoseiulus californicus & & & & & 2 & 0,05 & 2 & 0,005 \\
\hline & & Neoseiulus tunus & 2 & 0,04 & 11 & 0,03 & & & 13 & 0,03 \\
\hline & & Typhlodromalus aripo & 2 & 0,04 & 51 & 0,18 & 23 & 0,6 & 76 & 0,2 \\
\hline & & Typhlodromina tropica & 1 & 0,02 & & & 1 & 0,02 & 2 & 0,005 \\
\hline & & Typhlodromips mangleae & 16 & 0,3 & & & 6 & 0,1 & 22 & 0,06 \\
\hline Oribatida & & & 2 & 0,04 & 80 & 0,3 & 1 & 0,02 & 83 & 0,2 \\
\hline Total & & & 4.081 & 11.3 & 28.209 & 78,1 & 3.804 & 10,5 & 36.094 & \\
\hline
\end{tabular}


Tetranychidae representou $13,8 \%$ dos ácaros, sendo Neotetranychus asper Feres \& Flechtmann, 2000 o mais abundante $(13,4 \%)$, sendo mais comum na cultivar Caigangue. Neotetranychus asper foi descrito em Alchornea glandulosa (Euphorbiaceae), no estado de São Paulo. São conhecidas atualmente apenas nove espécies do gênero Neotetranychus Trägardh, 1915, sendo duas delas relatadas em Rubus sp. (Bolland et al., 1998; Migeon \& FlechtMAnN, 2004). No Brasil, quatro espécies desse gênero são conhecidas e nenhuma delas relatadas nesta cultura (Feres \& Flechtmann, 2000). Eotetranychus sp., foi o segundo tetraniquídeo mais abundante, não sendo observado na cultivar Tupy.

Oligonychus yothersi (McGregor, 1914), encontrado em baixas populações nas cultivares avaliadas, é conhecido como ácaro vermelho da ervamate (Ilex paraguariensis, Aquifoliaceae). Esta espécie ataca folhas novas e maduras da erva-mate, provocando bronzeamento ou, em ataques severos, queda de folhas (Coll \& Saini, 1992; Penteado, 1995; Ferla et al., 2005). Entretanto, no presente estudo não foram observados sinais de bronzeamento das folhas. Possivelmente a presença desta espécie nesta cultura se deve à migração deste ácaro da erva-mate de campos de cultivo adjacentes para a amora-preta, pois nesta região a erva-mate é cultivada de forma intensiva.

Brevipalpus phoenicis (Geijskes, 1939), o único Tenuipalpidae registrado, representou apenas $0,3 \%$ do total de ácaros coletados, sendo mais abundante na cultivar Caigangue. Brevipalpus phoenicis é uma espécie polífaga e amplamente distribuída no mundo, causando danos econômicos em diversas plantas (BAKER \& TUTTLE, 1987). É referido como ácaro da leprose dos citros, pois é vetor do vírus que causa essa doença nos citros (Chiavegato, 1987). Também está associado à manchaanular do cafeeiro (ChAGAS, 1973) e no maracujazeiro atua como vetor da pinta-verde (KiтAJIMA et al., 1997).

Cinco famílias de ácaros predadores foram coletadas neste estudo, sendo Phytoseiidae e Stigmaeidae as mais constantes (presentes em mais de 50\% das coletas). Embora os ácaros fitófagos tenham sido mais abundantes, a riqueza de espécies foi maior no grupo de ácaros predadores. Os Stigmaeidae foram os mais abundantes, com cerca de $2 \%$ do total coletado. O único Stigmaeidae registrado foi Agistemus brasiliensis Matioli, Ueckermann \& Oliveira, 2002, sendo mais comumente encontrado na cultivar Caigangue, onde foram observadas maiores populações de Chakrabartiella sp. Espécies do gênero Agistemus Summers, 1960 tem sido relatadas como importantes inimigos naturais de ácaros fitófagos (MORAES, 2002), principalmente os Eriophyoidea (Thistlewood et al., 1996). Para alguns estigmeídeos, os eriofiídeos são preferidos como recurso alimentar na falta dos tetraniquídeos (Santos, 1991). Ferla \& Moraes (2003) avaliaram a biologia de Agistemus floridanus Gonzalez, 1965 e verificaram maior oviposição quando alimentado com Calacarus heveae Feres, 1992 (Eriophyidae) e Tenuipalpus heveae Baker, 1945 (Tenuipalpidae). Algumas espécies de Agistemus são de importância no controle de tetraniquídeos (COLLYER, 1964; LoRENZATO et al., 1986; Ferla \& Moraes, 1998).

Apenas $0,4 \%$ dos ácaros coletados pertenceram à família Phytoseiidae. Contudo, esta família apresentou maior riqueza, com 11 espécies. Comumente a riqueza de fitoseídeos são grandes em ambientes naturais, urbanos ou em agroecossistemas (Ferla \& Moraes, 2002a, b; Collier et al., 2004; Daud \& Feres, 2005). Durante o estudo, três espécies foram mais abundantes: Typhlodromalus aripo DeLeon, 1967 (0,2\%), T. mangleae DeLeon, 1967 (0,06\%) e Euseius ho (DeLeon, 1965) $(0,05 \%)$. Typhlodromalus aripo foi coletado em maior número em Caigangue e possivelmente suas populações estavam associadas à presença de $N$. asper e $O$. yothersi. Typhlodromalus aripo demonstrou controlar de forma efetiva as populações de Manonychellus tanajoa (Bondar, 1938) (Tetranychidae) após a sua introdução no continente africano (HANNA \& TOKO, 2001; YANYNEK \& HANNA, 2003).

Acaridae, Tarsonemidae e Tydeidae com hábitos alimentares variados foram observados em números muito baixos, exceto Tydeidae (1,4\% do total), sendo a maioria pertencente ao gênero Tydeus Koch, 1835. Maior abundância foi observada na cultivar Tupy. Ácaros da família Tydeidae alimentam-se de fungos, pólen e outros ácaros (KRANTZ \& LiNDQUIST, 1979) e podem ser presas alternativas de outros predadores, principalmente de fitoseídeos (BAKER, 1970).

Os menores valores dos índices foram observados na cultivar Caigangue (Tab. II). Nesta cultivar Chakrabartiella sp. foi mais abundante que as demais espécies. Resultados semelhantes aos observados neste estudo, com diversidade abaixo de 50\% (Tab. II), foram observados na cultura da seringueira (Feres et al., 2002). A baixa diversidade observada neste estudo parece ser tendência em ecossistemas com altos teores de nutrientes ou enriquecidos e o aumento da produtividade aumenta a dominância e reduz a diversidade, enquanto que o aumento da diversidade de plantas aumenta significativamente a produtividade de agroecossistemas (Tilman et al., 1996; Odum, 2008). Além disso, o enriquecimento por nutrientes pode afetar a composição das espécies (Tilman, 1987) trazendo ervas daninhas nocivas, pragas exóticas e organismos transmissores de doenças (adaptados a ambientes com altos teores de nutrientes).

Flutuação populacional. Na cultivar Brazos, Chakrabartiella sp. apresentou pico populacional no mês de agosto nos dois anos avaliados, sendo menos

Tabela II. Parâmetros observados nas cultivares Brazos, Caigangue e Tupy de amora-preta (Rubus fruticosus, Rosaceae), no município de Ilópolis, Rio Grande do Sul ( $\mathrm{R}$, índice da riqueza das espécies de Margalef; H', Shannon-Wiener; H max, diversidade máxima teórica; E, equitabilidade de Pielou).

\begin{tabular}{lccl}
\hline & Brazos & Caigangue & Tupy \\
\hline $\mathrm{R}$ & 2,04 & 1,65 & 2,3 \\
H' & 1,14 & 0,42 & 1,29 \\
H $\max$ & 2,87 & 2,88 & 2,97 \\
E & 0,39 & 0,14 & 0,43 \\
\hline
\end{tabular}


evidente em 2003 (Fig. 2), períodos onde foram observadas diminuição da umidade relativa do ar e da precipitação (Fig. 3). Os tetraniquídeos foram registrados durante todo o estudo e maiores populações foram observadas em outubro de 2002, coincidindo com alta precipitação e umidade relativa do ar. Agistemus brasiliensis foi a espécie predadora mais abundante, com picos populacionais de fevereiro a julho de 2002, período em que foram observadas populações expressivas de $N$. asper e Chakrabartiella sp. Contudo, a diminuição das populações destes predadores aconteceu em agosto de 2002, quando foram observadas as maiores populações de Chakrabartiella sp. Além disso, também foram observadas altas populações em janeiro e fevereiro de 2003, coincidindo com o aumento das populações de $N$. asper. Devido à diversidade de presas observadas durante o período com altas populações de A. brasiliensis, esta espécie possivelmente se mantenha abundante nesta

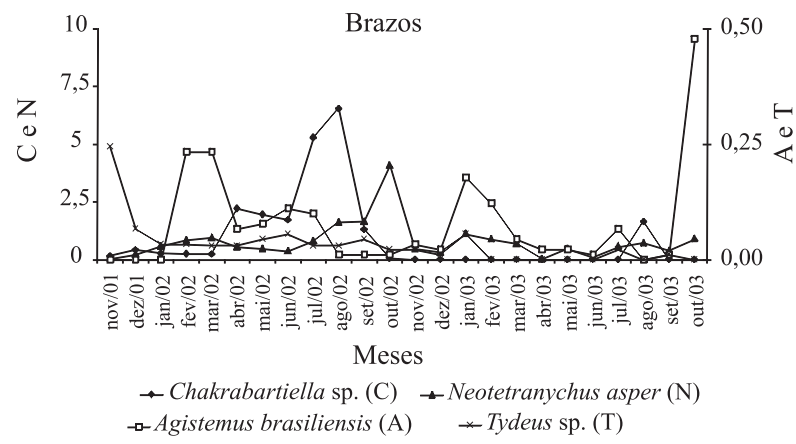

Caigangue
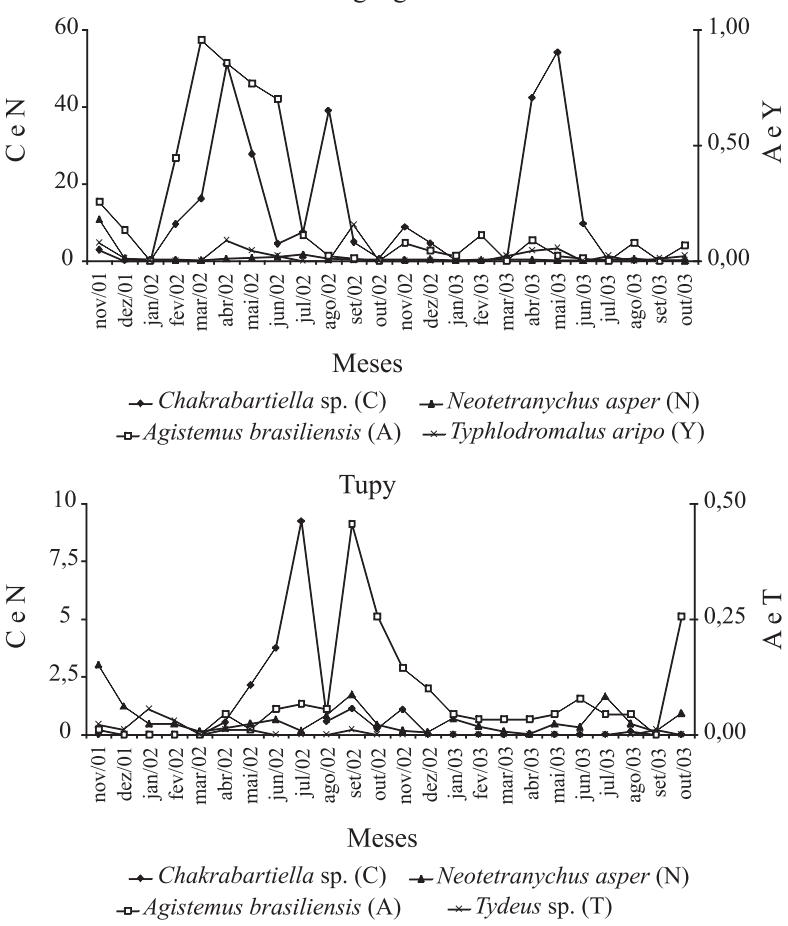

Figura 2. Flutuação populacional das principais espécies acarinas (média de ácaros/folíolo) nas cultivares Brazos, Caigangue e Tupy de amora preta (Rubus fruticosus, Rosaceae), no município de Ilópolis, Rio Grande do Sul, entre os meses de novembro de 2001 e outubro de 2003 (A, Agistemus brasiliensis; C, Chakrabartiella sp.; N, Neotetranychus asper; T, Tydeus sp.; Y, Typhlodromalus aripo). cultura por aceitar uma maior diversidade de alimentos. Agistemus floridanus também demonstrou aceitação a uma grande diversidade de alimentos em seringueira (Ferla \& Moraes, 2003). O pico populacional de Tydeus sp. foi observado em novembro de 2002, mas suas populações mantiveram-se constantes até janeiro de 2003.

$\mathrm{Na}$ cultivar Caigangue foram observados três picos populacionais de Chakrabartiella sp. nos meses de abril e agosto de 2002 e abril e maio de 2003. O aumento populacional deste grupo coincidiu com menores índices de precipitação e umidade relativa do ar. Durante todo o período avaliado, as populações de Neotetranychus asper mantiveram-se baixas. Maiores populações de $A$. brasiliensis foram observadas concomitantemente a expressivas populações de Chakrabartiella sp., principalmente entre os meses de fevereiro e junho de 2002. Typhlodromalus aripo também foi mais abundante nos meses em que foram observadas maiores populações de diptilomiopídeos, principalmente nos meses de abril e setembro de 2002 e abril e maio de 2003.

$\mathrm{Na}$ cultivar Tupy, maior índice populacional de Chakrabartiella sp. foi observado em julho de 2002, período de diminuição da umidade relativa do ar. Neotetranychus asper teve maior índice nos meses de novembro de 2001, setembro de 2002 e julho de 2003 , períodos nos quais foi observada a diminuição da umidade relativa do ar. Agistemus brasiliensis esteve presente, em altas populações, nos períodos em que os tetraniquídeos e diptilomiopídeos foram mais abundantes.

Segundo Raseira et al. (1984) as cultivares avaliadas neste estudo apresentam diferentes produtividade, morfologia e perfilhamento. Além disso, a frutificação ocorre em períodos diferentes e seus frutos apresentam sabor, acidez e açúcar distintos. Estas características podem influenciar a presença de populações de ácaros herbívoros e por isso algumas cultivares podem ser mais susceptíveis à fauna acarina que outras. Neste estudo, a cultivar Caigangue demonstrou ser mais suscetível, pois apresentou populações de Chakrabartiella sp. e Neotetranychus asper maiores que as demais cultivares. Este fenômeno também foi observado com fitoseídeos, pois a textura da folha influenciou a performance de predação de

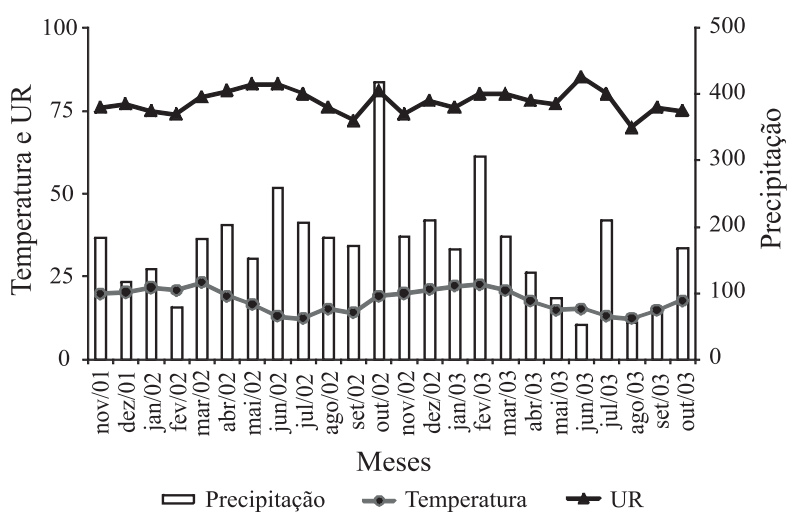

Figura 3. Parâmetros climáticos da Estação Climatológica da Embrapa Uva e Vinho, município de Bento Gonçalves, Rio Grande do Sul, entre os meses de novembro de 2001 a outubro de 2003. 
Phytoseius plumifer (Canestrini \& Fanzago, 1876) e Euseius gossipy (El-Badry, 1967) (RASMY \& El BANHAWAY, 1974; RASMY, 1977).

Prevalência de espécies de tetraniquídeos e ácaros predadores. Em relação aos tetraniquídeos, $N$. asper foi, de maneira geral, a espécie mais abundante neste estudo (Fig. 4). A segunda espécie mais numerosa foi Eotetranychus sp., cuja frequência foi maior na cultivar Brazos, entre os meses de dezembro de 2001 e março de 2002 e junho de 2003; na cultivar Caigangue foi mais frequente entre fevereiro e abril de 2002 e julho, agosto e outubro de 2003. Esta espécie não foi observada na cultivar Tupy. Oligonychus yothersi e Tetranychus mexicanus (McGregor, 1950) foram encontradas em menor frequência, sendo T. mexicanus observada apenas na cultivar Caigangue.

Agistemus brasiliensis foi a espécie de predador mais numerosa nas três cultivares avaliadas durante quase todo o estudo (Fig. 5). Cunaxoides sp. foi mais frequente nos meses de novembro e dezembro de 2001 nas cultivares Brazos e Tupy e em abril, agosto, setembro e dezembro de 2002 na cultivar Brazos. Typhlodromalus aripo foi mais frequente na cultivar Caigangue nos meses de setembro de 2002, março, maio, julho e setembro de 2003; na cultivar Tupy, também foi observada nos meses

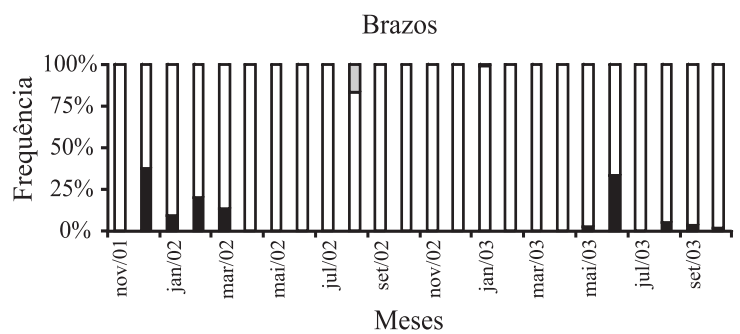

[Eotetranychus sp. ㅁeotetranychus asper $\mathbf{\square}$ Oligonychus yothersi Caigangue

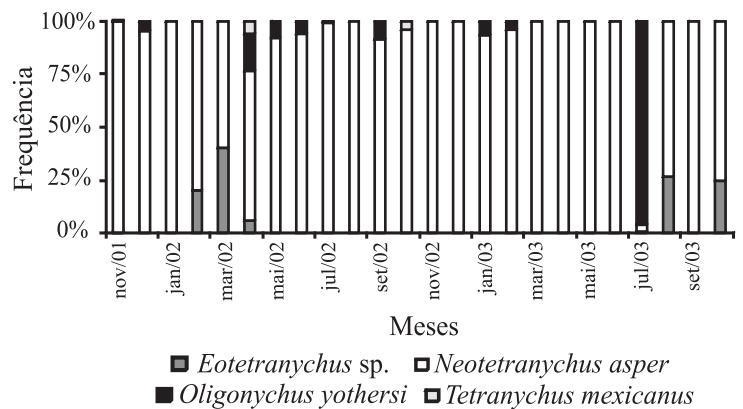

Tupy

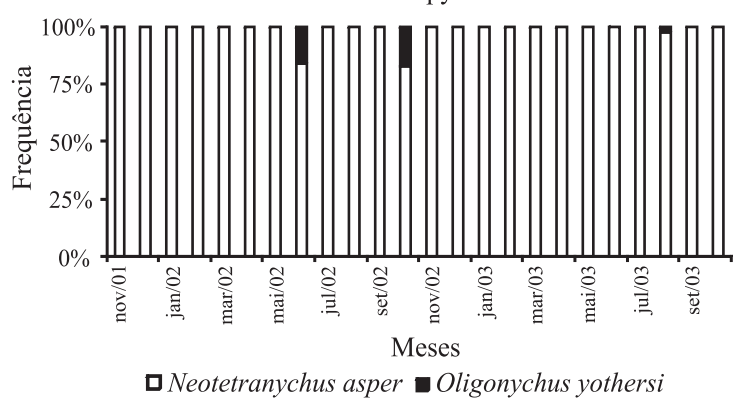

Figura 4. Frequência de Tetranychidae nas cultivares Brazos, Caigangue e Tupy de amora-preta (Rubus fruticosus, Rosaceae), em Ilópolis, Rio Grande do Sul, entre os meses de novembro de 2001 a outubro de 2003. de maio, julho e dezembro de 2002 e fevereiro e março de 2003; na cultivar Brazos foi observada apenas em novembro de 2001.

Dentre os predadores, Agistemus brasiliensis destacou-se com maiores populações e maior persistência durante o período de avaliação. Esta espécie produziu ovos quando se alimentou de Panonychus citri McGregor, 1916 e Brevipalpus phoenicis Geijskes, 1939 e sobreviveu alimentando-se de Tetranychus urticae Koch, 1836. A taxa reprodutiva aumentou quando foi acrescentado pólen de Typha sp. (Typhaceae) (Matiol et al., 2002). Grandes populações de Agistemus brasiliensis foram observadas associadas às populações de eriofídeos em erva-mate (FerLa et al., 2007). Agistemus floridanus Gonzalez, 1965 demonstrou alimentar-se de pólen, tetraniquídeos, tenuipalpídeos e eriofídeos (FERLA \& MORAES, 2003).

Typhlodromalus aripo também foi a espécie mais frequente na avaliação da acarofauna associada à cultura do morango e plantas do entorno, no estado do Rio Grande do Sul (FerLa et al., 2007). É a espécie de predador comumente associada à Mononychellus tanajoa (Bondar, 1938) na cultura da mandioca no Nordeste do Brasil (Moraes et al., 1991).

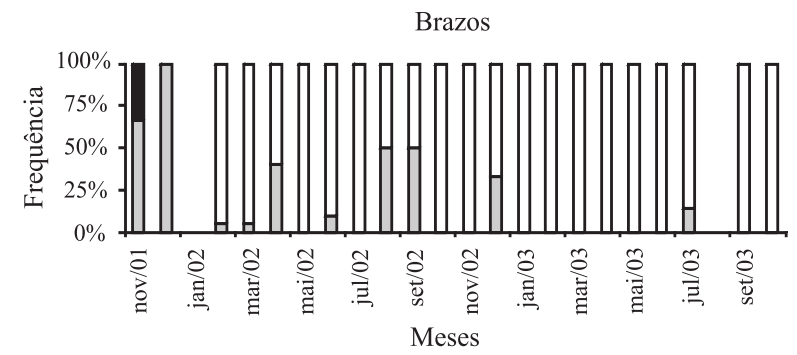

口Cunaxoides sp. \} \text { Agistemus brasiliensis } \mathbf { \square } \text { Typhlodromalus aripo } Caigangue

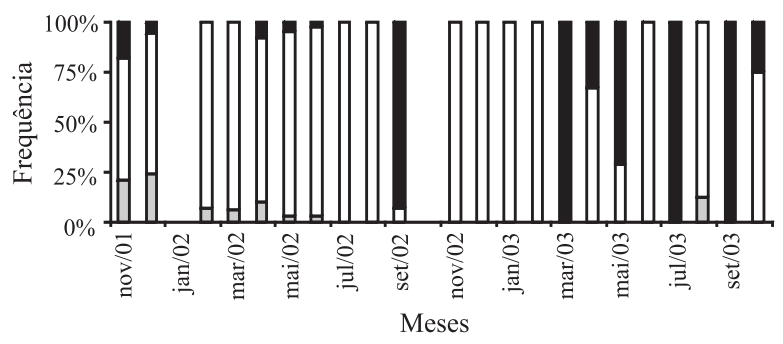

$\mathbf{\square}$ Cunaxoides sp. $\mathbf{\square}$ Agistemus brasiliensis $\boldsymbol{\square}$ Typhlodromalus aripo

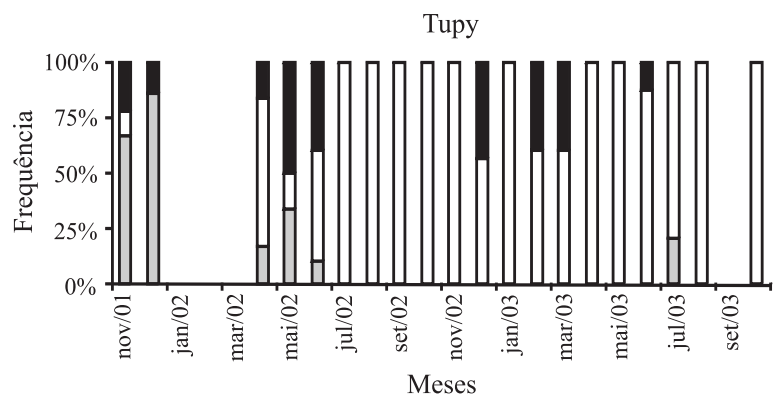

$\mathbf{\square}$ Cunaxoides sp. $\mathbf{\square}$ Agistemus brasiliensis $\mathbf{\square}$ Typhlodromalus aripo

Figura 5. Frequência de ácaros predadores nascultivares Brazos, Caigangue e Tupy de amora-preta (Rubus fruticosus, Rosaceae), em Ilópolis, Rio Grande do Sul, entre os meses de novembro de 2001 a outubro de 2003. 
Agradecimentos. Ao Museu de Ciências Naturais (MCN/ UNIVATES) e ao Centro Universitário UNIVATES pelo financiamento do projeto.

\section{REFERÊNCIAS BIBLIOGRÁFICAS}

Amrine JR., J. W. \& Manson, D. C. M. 1996. Preparation, mounting and descriptive study of eriophyoid mites. In: LINDQUIST, E. E.; SABELIS, M. W. \& BRUIN, J. eds. World crop pests: eriophyoid mites their biology, natural enemies and control. Amsterdam, Elsevier. p.383-396.

Amrine Jr., J. W. \& Stasny, T. A. 1994. Catalog of the Eriophioidea (Acarina: Prostigmata) of the world. Bloomfield, Indira Publishing House. 798p.

Antunes, L. E. C.; Regina, M. A. \& Duarte Filho, J. 2002. A cultura da amora preta. Belo Horizonte, EPAMIG. (Boletim Técnico, 69). 28p.

BAKER, E. W. 1970. The genus Tydeus: subgenera and species groups with descriptions of new species (Acari: Tydeidae). Annals of the Entomological Society of America 63(1):163-177.

Baker, E. W. \& Tuttle, D. M. 1987. The false spider mites of Mexico (Tenuipalpidae: Acari). Florida, USDA Agricultural Research Service. (Technical Bulletin, 1706). 237p.

Bassols, M. DO C. 1980. A cultura da amora preta. Pelotas, EMBRAPA-UEPAE Cascata, (EMBRAPA-UEPAE Cascata. Circular Técnica, 4). 11p.

Bolland, H. R.; Gutierrez, J. \& Flechtmann, C. H. W. 1998. World catalogue of the spider mite family (Acari: Tetranychidae). Leiden, Brill. 392p.

Chagas, C. M. 1973. Associação do ácaro Brevipalpus phoenicis (Geijskes) à mancha anular do cafeeiro. O Biológico 39:229-232.

Chiavegato, L. G. 1987. A leprose do citros no estado de São Paulo. Revista Laranja 2(1):7-18.

CoLL, O. R. DE \& SAINI, E. D. 1992. Insectos y acaros perjudiciales al cultivo de la yerba mate en la Republica Argentina. Montecarlo, INTA. 48p.

COLLYER, E. 1964. Phytophagous mites and their predators in New Zeland orchards. Journal of Agricultural Research 7:551-568.

Collier, K. F. S.; Lima, J. O. G. De \& Albuquerque, G. S. 2004. Predacious mites in papaya (Carica papaya L.) orchards: in search of a biological control agent of phytofagous mite pests. Neotropical Entomology 33(6):799-803.

DAuD, R. D. \& FERES, R. J. F. 2005. Diversidade e flutuação populacional de ácaros (Acari) em Mabea fistulifera Mart. (Euphorbiaceae) de dois fragmentos de mata estacional semidecídua em São José do Rio Preto, SP. Neotropical Entomology 34(2):191-201.

De Lillo, E. \& Duso, C. 1996. Currants and berries. In: Lindquist, E. E.; SABElis, M. W. \& Bruin, J. eds. Eriophyoid mites. Amsterdam, Elsevier. p.583-591.

Feres, R. J. F. \& Flechtmann, C. H. W. 2000. Four new Neotetranychus Trägardh (Acari: Tetranychidae) from São Paulo State. Acarologia 41(1-2):215-226.

Feres, R. J. F.; Rossa-Feres, D. de C.; Daud, R. D. \& Santos, R. S. 2002. Diversidade de Ácaros (Acari, Arachnida) em seringueiras (Hevea brasiliensis Muell. Arg., Euphorbiaceae) na região noroeste do estado de São Paulo, Brasil. Revista Brasileira de Zoologia 19(1):137-144.

Ferla, N. J.; Marchetti, M. M. \& Gonchalves, D. 2007. Ácaros predadores (Acari) associados à cultura do morango (Fragaria sp., Rosaceae) e plantas próximas no Estado do Rio Grande do Sul. Biota Neotropica 7(2):1-8.

Ferla, N. J.; Marchetti, M. M. \& Siebert, J. C. 2005. Acarofauna (Acari) de erva-mate (Ilex paraguariensis St. Hil.: Aqüifoliaceae) no Estado do Rio Grande do Sul. Biociências 13(2):133-142.

FerLa, N. J. \& Moraes, G. J. DE. 1998. Ácaros predadores em pomares de maçã no Rio Grande do Sul. Anais da Sociedade Entomológica 27(4):649-654.

2002a. Acaros (Arachnida, Acari) da seringueira (Hevea brasiliensis Muell. Arg.) no estado do Mato Grosso. Revista Brasileira de Zoologia 19(3):867-888. 2002b. Ácaros predadores (Acari) em plantas nativas e cultivadas do estado do Rio Grande do Sul. Revista Brasileira de Zoologia 19(4):1011-1031.

2003. Oviposição dos ácaros predadores Agistemus floridanus Gonzalez, Euseius concordis (Chant) e Neoseiulus anonymus (Chant \& Baker) (Acari) em resposta a diferentes tipos de alimento. Revista Brasileira de Zoologia 20(1):153-155.

Flechtmann, C. H. W. 1979. Ácaros de importância agrícola. São Paulo, Nobel. 189p
Hanna, R. \& Toko, M. 2001. Cassava green mite biological control in Africa: project overview and summary of progress. In: HANNA, R. \& Токо, M. eds. Proceedings of the Regional Meeting of the cassava Green Mite Biocontrol Project, Dar es Salaam, Tanzania, 15-17 November 2000. Cotonou, IITA. p.4-22.

Jeppson, L. R.; Keifer, H. H. \& Baker, E. W. 1975. Mites injurious to economic plants. Berkeley, University of California. $614 \mathrm{p}$.

Kitajima, E. W.; Rezende, J. A. M.; Rodrigues, J. C. V.; Chiavegato, L. G.; Piza JR., C. T. \& MorozinI, W. 1997. Green spot of passion fruit, a possible viral disease associated with infestation by the mite Brevipalpus phoenicis. Fitopatologia Brasileira 22(4):555-559.

KRANTZ, G. W. \& Lindquist, E. E. 1979. Evolution of phytophagous mites (Acari). Review Entomology 66(4):709-717.

Lorenzato, D.; Grellmann, E. O.; Chouene, E. C. \& Meyer-Cachapuz, L. M. 1986. Flutuação populacional de ácaros fitófagos e seus predadores associados à cultura de macieira (Malus domestica Bork) e efeitos dos controles químico e biológico. Agronomia Sulriograndense 22:135-151.

Matioli, A. L.; Ueckermann, E. A. \& Oliveira, C. A. L. 2002. Some stigmaeid and eupalopsellid mites from citrus orchards in Brazil (Acari: Stigmaeidae and Eupalopsellidae). International Journal of Acarology 28(2):99-120.

Migeon, A. \& Flechtmann, C. H. W. 2004. First additions and corrections to the world catalogue of the spider mite family (Acari: Tetranychidae). International Journal of Acarology 30(2): $143-152$

MoraEs, G. J. DE. 2002. Controle biológico de ácaros fitófagos com ácaros predadores. In: Parra, J. R. P.; Botelho, P. S. M.; CorreÂFerreira, B. S. \& Bento, J. M. S. eds. Controle biológico no Brasil: parasitóides e predadores. Barueri, Manole. p.225-237.

Moraes, G. J. De; Mesa N. C. \& Braun, A. 1991. Some Phytoseiid mites of Latin América (Acari: Phytoseiidae). International Journal of Acarology 17(2):117-139.

Odum, E. P. 2008. Fundamentos de ecologia. São Paulo, Cengage Learning. 612p.

OldFieLD, G. N. 1996. Diversity and host specificity. In: LinDQUIST, E. E.; Sabelis, M. W. \& Bruin, J. eds. World crop pest eriophyoids mites: their biology, natural enemies and control. Amsterdam, Elsevier. p.199-216.

Penteado, S. R. 1995. Principais pragas da erva-mate e medidas alternativas para o seu controle. In: Winge, H.; FerReIRA, A. G.; Mariath, J. E. DE A. \& TARASConi, L. C. eds. Erva-mate: biologia e cultura no Cone Sul. Porto Alegre, UFRGS, p.109-120.

Raseira, M. do C. B.; Santos, A. M. dos \& Madall, J. C. M. 1984 Amora preta: cultivo e utilização. Pelotas, EMBRAPACNPFT, (Informativo, 23). 20p.

Rasmy, A. H. 1977. Predatory efficiency and biology of the predatory mite, Amblyseius gossipi (Acarina: Phytoseiidae) as affected by plant surfaces. Entomophaga 22:421-423.

Rasmy, A. H. \& El Banhawy, E. M. 1974. Behaviour and bionomics of the predatory mite Phytoseius plumifer (Acarina: Phytoseiidae) as affected by physical surface features of host plants. Entomophaga 19:255-257.

Rodrigues, W. C. 2005. DivEs - Diversidade de espécies. Versão 2.0. Software e Guia do Usuário.. Disponível em: <http://www.ebras.bio.br/dives>. Acesso em: 22.04.2005.

Santos, M. A. 1991. Searching behavior and association response of Zetzellia mali (Acarina: Stigmaeidae). Experimental \& Applied Acarology 11:81-87.

Thistlewood, H. M. A.; Clements, D. R. \& Harmsen, R. 1996 Stigmaeidae. In: Lindouist, E. E.; S Abelis, M. W. \& Bruin, J. eds. Eriophyoid mites their biology, natural enemies and control. Amsterdam, Elsevier. p.457-470

TiLman, D. 1987. Secondary succession and the pattern of plant dominance along experimental nitrogen gradients. Ecological Monographs 57:189-214.

Tilman, D.; Wedin, D. \& Knops, J. 1996. Productivity and sustainability influenced by biodiversity in grassland ecosystems. Nature 379:718-720.

YANineK, J. S. \& Hanna, R. 2003. Cassava green mite in Africa: unique example of successful classical biological control of a mite pest on a continental scale. In: NeuEnschwander, P. Borgemeister, C. \& Langewald, L. eds. Biological control in IPM systems in Africa. Wallingford, UK, CABI. p.61-75.

ZhANG, Z. Q. 2003. Mites of greenhouses: identification, biology and control. Cambridge, CABI. 244p. 